\title{
Primary flexor enthesopathy of the canine elbow: imaging and arthroscopic findings in eight dogs with discrete radiographic changes
}

B. Van Ryssen'; E. de Bakker; Y. Beaumlin'; Y. C. A. Samoy'; D. Van Vynckt'; I. Gielen"; R. Ducatelle'; H. van Bree ${ }^{1}$

${ }^{1}$ Ghent University, Faculty of Veterinary Medicine, Department of Veterinary Diagnostic Imaging and Small Animal Orthopaedics, Merelbeke, Belgium; ${ }^{2}$ Ghent University, Faculty of Veterinary Medicine, Department of Veterinary

Pathology, Merelbeke, Belgium

\section{Keywords}

Elbow, dog, medial epicondyle, flexor enthesopathy, diagnosis

\section{Summary}

Objective: To describe the radiographic, ultrasonographic, computed tomography (CT), magnetic resonance imaging (MRI), and arthroscopic findings in eight dogs with elbow lameness caused by primary flexor enthesopathy.

Study design: Clinical study.

Animals: Eight client-owned dogs.

Methods: In all dogs, lameness was localized to the elbow by clinical examination. Radiographic examination, ultrasound, CT and MRI were performed prior to arthroscopy. In seven dogs, surgical treatment and subsequent histopathology were performed.

Results: Primary enthesopathy of the medial epicondyle was diagnosed in eight dogs (13

Correspondence to:

Prof. Bernadette Van Ryssen

Ghent University

Faculty of Veterinary Medicine

Medical Imaging and Small Animal Orthopaedics

Salisburylaan 133

Merelbeke, 9820

Belgium

Phone: +32 92647650

E-mail: Bernadette.VanRyssen@UGent.be joints) by combining the minimal radiographic changes with specific ultrasonographic, CT, MRI and arthroscopic findings at the medial epicondyle. In all joints, any other pathology could be excluded. Histopathology of the affected tissue revealed degeneration and metaplasia in the flexor muscles.

Conclusions: Primary flexor enthesopathy at the medial epicondyle is an unrecognized condition and is a possible cause of elbow lameness in the dog. Diagnosis is based on specific imaging and arthroscopic findings.

Clinical relevance: The most important cause of elbow lameness in dogs is medial coronoid disease. Often this condition presents with minimal radiographic and arthroscopic changes. In these cases, primary enthesopathy of the medial epicondyle should be considered as a differential diagnosis, in order to make the correct treatment decision.

Vet Comp Orthop Traumatol 2012; 25: 239-245 doi:10.3415/VCOT-11-03-0038 Received: March 9, 2011

Accepted: December 4, 2011

Pre-published online: March 26, 2012

\section{Introduction}

The elbow is a common location for thoracic limb lameness in medium and large breed dogs. The most frequent diagnosis is fragmented coronoid process, based on the history and the clinical and radiographic examination. Typical radiographic findings are fragmentation, deformation or an unclear outline of the medial coronoid process, often accompanied by sclerosis or secondary osteoarthritis (1). However, in a considerable number of cases, diagnosis of fragmented coronoid process is chall- enging because of minimal radiographic changes (2-5).

Ununited medial epicondyle has been reported as a rare problem and is often considered as a clinically insignificant finding (6). It was originally reported in 1966 after which several clinical reports were written, discussing and disagreeing on the terminology, origin, clinical significance and prevalence of this condition (7-15). Most reports described the condition as a separate bony fragment, an avulsed fragment, or a calcification of the flexor muscles. A German report from 2004 described the radiographic and arthroscopic findings in 26 joints ( 23 dogs) with a calcification ranging in size from $5 \times 3 \mathrm{~mm}$ to $30 \times 12 \mathrm{~mm}$ that was mainly visible on the craniocaudal view (16). One report described a 'spur' at the caudal edge of the medial epicondyle as the only finding and suggested that pathology of the flexor muscles might be present without radiographic changes at an early stage (17). This disorder was called enthesopathy because of the pathological changes within the flexor-bone connection, called the enthesis $(18,19)$.

A review of the recent publications and orthopaedic handbooks illustrated that ununited medial epicondyle receives little attention as a cause of elbow lameness and an ununited medial epicondyle is not listed on the list of differential diagnoses (6). Attention is exclusively given to fragmented coronoid process, osteochondritis dissecans of the humeral condyle, ununited anconeal process and incongruity $(20,21)$. One recent report described the coincidental diagnosis of ununited medial epicondyle and elbow osteoarthritis in a litter of Labrador dogs and suggested a higher inci- 
Table 1 Information on the breed, sex and age distribution as well as the uni- or bilateral lesions and treatment in eight dogs affected by primary flexor enthesopathy of the elbow.

\begin{tabular}{|c|c|c|c|c|c|}
\hline Case & Breed & Sex & Age & Lesions & Treatment \\
\hline 1 & Samoyed & Male & 5 years & Bilateral & Bilateral \\
\hline 2 & Mixed breed & Male & 7 years & Unilateral & Unilateral \\
\hline 3 & Border Collie & Female & 3.5 years & Bilateral & Bilateral \\
\hline 4 & Boerboel & Male & 2 years & Bilateral & Unilateral \\
\hline 5 & Bernese Mountain dog & Female & 3.5 years & Unilateral & Unilateral \\
\hline 6 & Bernese Mountain dog & Female & 3 years & Bilateral & Unilateral \\
\hline 7 & $\begin{array}{l}\text { Great Swiss } \\
\text { Mountain dog }\end{array}$ & Male & 3.75 years & Unilateral & Unilateral \\
\hline 8 & $\begin{array}{l}\text { Great Swiss } \\
\text { Mountain dog }\end{array}$ & Male & 3.75 years & Bilateral & Bilateral \\
\hline
\end{tabular}

dence than presumed, although no clinical importance was attributed to the finding (22). Furthermore, an ununited medial epicondyle is no longer considered as a disorder belonging to the elbow dysplasia complex because of its low prevalence and low clinical impact (23). However in the author's experience, clinically significant lesions in the area of the medial epicondyle are diagnosed on a regular basis. They are not only visible as a clear fragment or calcification, but also with minimal radiographic lesions. In a subsequent series of 200 radiographic elbow images, changes of the medial epicondyle were seen in $40 \%$ of the cases (data being prepared for publication by the authors). A calcified body near the medial epicondyle was diagnosed

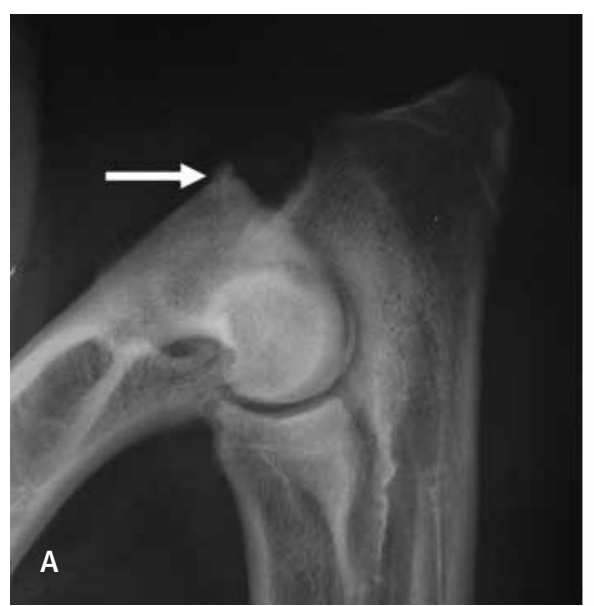

in five percent of the 200 elbows. In half of these cases, the calcified body was diagnosed as a sign of primary flexor enthesopathy. A spur or irregular outline of the medial epicondyle without the presence of a fragment was seen in nearly $25 \%$ of the 200 elbows. In most cases this was an expression of osteoarthritis, but in two percent of the total number of elbows, the changes were an expression of primary flexor enthesopathy.

This paper describes the discrete radiographic and specific ultrasonographic, computed tomography (CT), magnetic resonance imaging (MRI), and arthroscopic findings in eight dogs with elbow lameness, which were admitted because of a suspected fragmented coronoid process and were diagnosed with pathological changes of the medial epicondyle and the attaching flexor muscles.

\section{Material and methods}

Medical records of eight dogs evaluated and treated over a period of five years were analyzed. In all dogs, a complete history and results of a clinical examination were available as well as the radiographic, CT, and arthroscopic images (13 joints). In three dogs (five joints), additional ultrasound and MRI images were available. In case of surgical treatment, histopathology was carried out.

Clinical examination included inspection on walk and trot and palpation to define the range-of-motion, joint distension, and pain reaction. Detailed scoring of lameness was done by assignment of grades on a scale from zero to 10 , a system which has been described for equine lameness evaluation $(24,25)$.

Radiographic examination was performed with the dog sedated, and included three standard projections of both elbows: the mediolateral flexed and extended view and a $15^{\circ}$ oblique craniolateral-caudomedial view.

Ultrasonography of the medial aspect of the elbow was performed in three dogs (five joints) with a linear, $10-15 \mathrm{MHz}$ probe .

Computed tomography was performed for all patients using a helical $\mathrm{CT}^{\mathrm{b}}$ with 1

Fig. 1 Radiographic findings in three joints affected by primary flexor enthesopathy. A) Bernese Mountain dog, three-years-old, with a discrete spur on the medial condylar ridge of the humerus (arrow), mild osteoarthritis, and subtrochlear sclerosis (case 6). B) Boerboel, two-years-old, with a discrete spur (arrow) at the medial epicondyle (case 4). C) Great Swiss Mountain dog, 3.75-years-old, with sclerosis and a radiolucent area (arrow) at the medial epicondyle (case 8). 
mm slice thickness and a bone reconstruction window of both elbows.

Magnetic resonance imaging of the elbow was performed in three dogs (five joints) in transverse, sagittal and dorsal planes with a $0.2 \mathrm{~T}$, permanent magnet $^{\mathrm{c}}$ using T1-weighted, T2-weighted and STIR sequences.

Arthroscopy was performed with a 2.4 $\mathrm{mm}$ arthroscope ${ }^{\mathrm{d}}$ via a standard medial approach (26).

Treatment consisted of one single intraarticular administration of a corticosteroid (methylprednisolon $0.5 \mathrm{mg} / \mathrm{kg}$ ) combined with six weeks restricted exercise in one dog (two joints). Seven dogs (10 joints) were treated by partial removal of the affected muscle, followed by six weeks restricted exercise with walks on leash and administration of non-steroidal anti-inflammatory drugs ( 7 dogs, 10 joints). Broad spectrum antibiotic drugs were administered for five days postoperatively. Two affected contralateral joints were left untreated.

Treatment results were assessed with a questionnaire in which the owners answered questions regarding the lameness status, activity level, and need for medication and by a clinical examination three months to four years after treatment.

\section{Results}

The diagnosis of primary flexor enthesopathy was made in eight dogs. Five dogs were affected bilaterally. Breed, sex and age are listed in Table 1. Lameness had a gradual onset in five dogs and an acute onset after exercise without obvious trau$m a$ in three dogs. Non-steroidal antiinflammatory drugs were administered in all dogs resulting in a temporary improvement or no improvement at all.

On clinical examination the dogs showed a light to moderate lameness (grade 2 to 6 on a scale of 10), varying elbow distension and pain reaction on hyperextension and a full range-of-motion. In all cases, a firm, well-defined swelling could be pal-

\footnotetext{
MyLab 30: Esaote, Firenze, Italy

CT Prospeed: GE, Milwaukee, USA

Airis Mate: Hitachi, Tokyo, Japan

Richard Wolf GmbH, Knittlingen, Germany
}

Fig. 2

Ultrasound of the flexor muscles, longitudinal plane, of a Great Swiss Mountain dog, 3.75-yearsold, with pathological changes: moderate outwart bowing (broad arrow), local decreased echogenicity (narrow arrows), and thickening can be noticed (case 7).

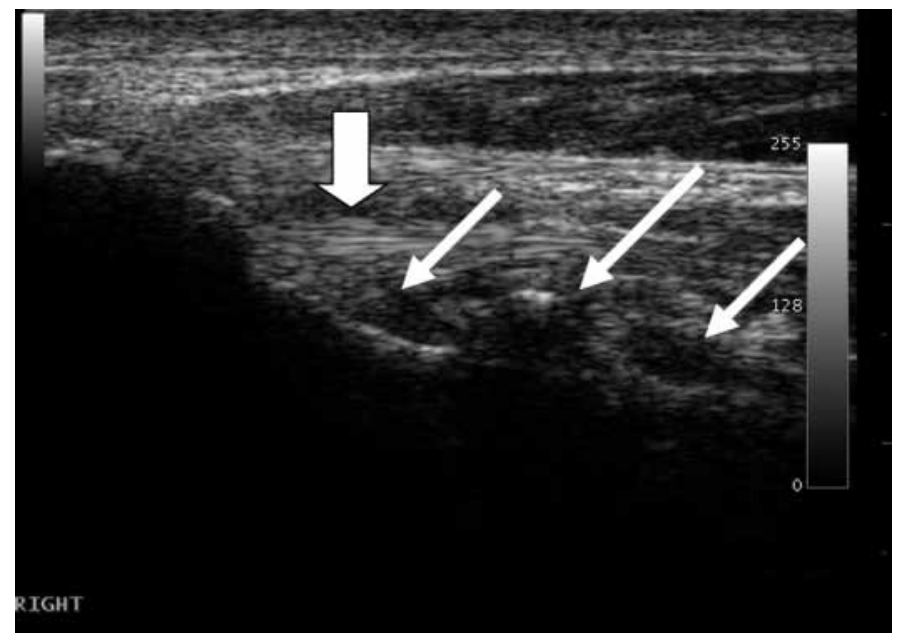

pated on the medial side of the elbow joint, caudodistal to the medial epicondyle. Pressure in that region was not particularly consistent in eliciting signs of pain.

In all 13 joints, discrete radiographic changes were detected. Radiographic changes of the medial epicondyle were seen unilaterally in three dogs and bilaterally in five dogs. The medial epicondyle showed a discrete spur in 11 joints ( Fig. $1 \mathrm{~A}, \mathrm{~B}$ ). In two joints of the same dog, the medial epicondyle was sclerotic with a small radiolucent area ( Fig. 1C). Radiographic signs of osteoarthritis were absent in twelve joints. One joint showed mild osteoarthritis (grade 1 based on the scale by the International Elbow Working Group). In all dogs, the medial coronoid process was well delineated and had a normal triangular shape. Subtrochlear sclerosis was absent in all joints, ex-
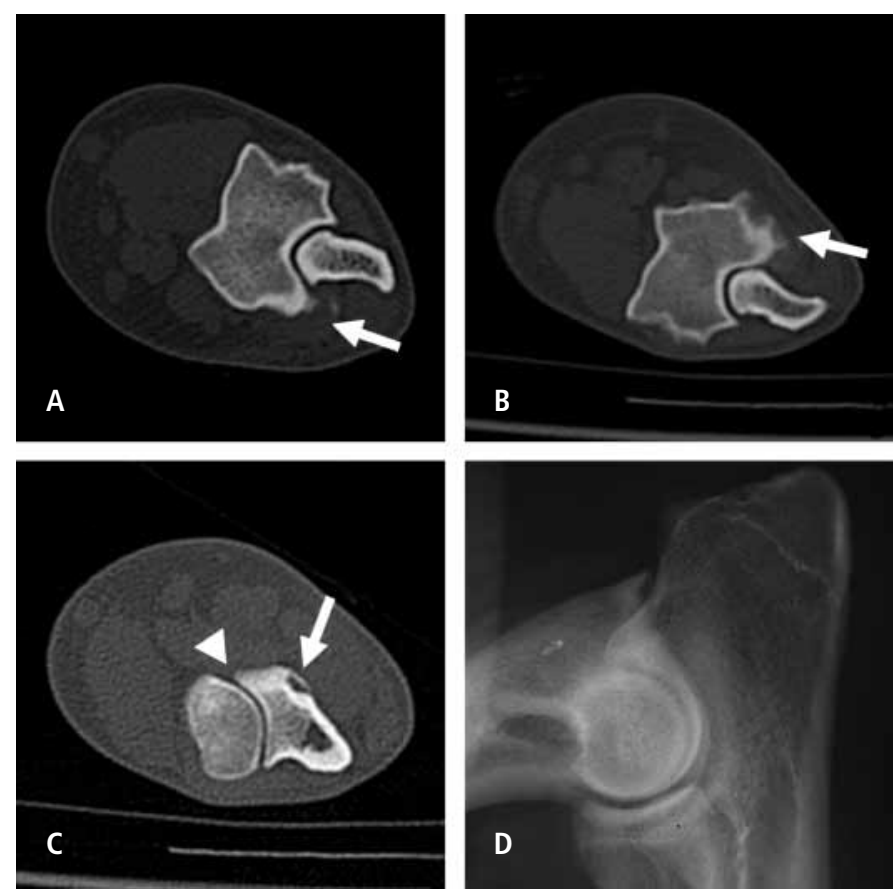

Fig. 3 Computed tomography transverse slices ( $A$ and $B$ at level of the humeral condyles, $C$ at level of the medial coronoid process) of a five-year-old Samoyed with bilateral lesions. A) Spur and sclerosis at the medial condyle (right elbow). B) Sclerosis and periosteal reaction at the medial epicondyle (left elbow). C) Intact medial coronoid process (arrow head), minimal osteoarthritis at the medial side of the ulna (arrow) (left elbow). D) Corresponding radiographic image of the left elbow (case 1). 
cept for the one joint with signs of mild osteoarthritis ( $>$ Fig. 1A). The medial part of the humeral condyle was round and smooth and there was no clear step or enlargement of the joint space suggesting the presence of incongruity. The absence of incongruity was confirmed by CT.

Ultrasound was performed in five affected joints (three dogs). A mild to moderate outward bowing of the flexor muscles and presence of fluid around and within the affected muscles was seen in all joints. The echogenicity of the flexors was decreased in four out of five joints and the flexors were thickened in three out of five joints ( Fig. 2).

Computed tomography was performed in all dogs. The medial epicondyle was sclerotic and showed a periosteal reaction in all affected joints ( Fig. 3 A, B). The medial
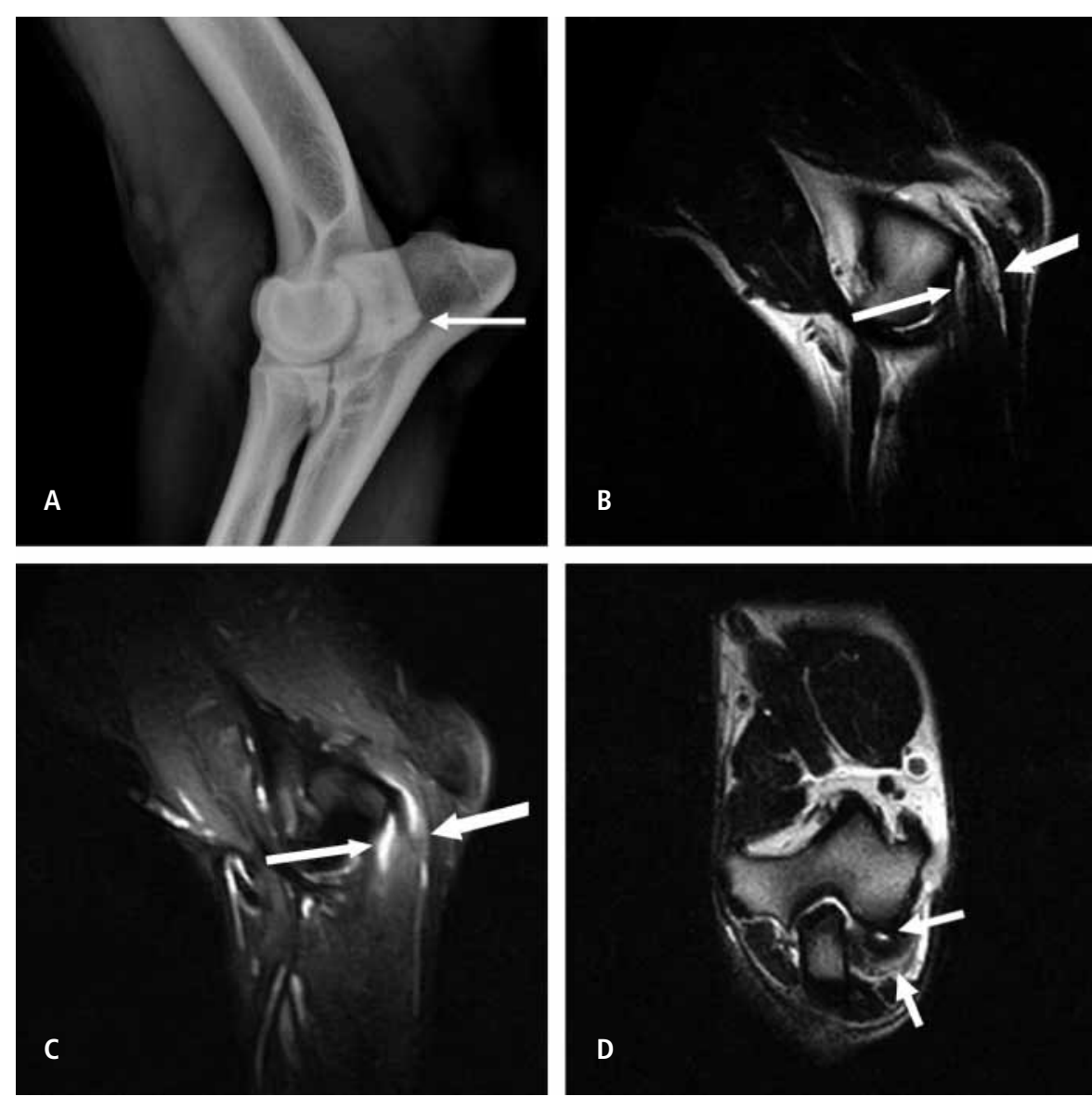

Fig. 4 Great Swiss Mountain dog, 3.5-years-old. A) Lateral extended radiographic view; minimal spur is indicated with an arrow. B - D are images from the magnetic resonance imaging examination. B) T2 weighted sagittal image; hyperintense fluid signal is present around the flexor tendons (arrows). C) STIR sagittal image; hyperintense fluid signal is present around the flexor tendons (arrows). D) T2 transverse image; hyperintense signal within the deep fibres of the flexor tendon origin (arrows), increased signal change of soft tissue (case 7).

coronoid process did not show any signs of subchondral bone cracks, fissures or fragmentation, which was subsequently confirmed during arthroscopic inspection ( Fig. 3 C). The medial aspect of the humeral condyle did not show any heterogenity or stripes as signs of kissing lesions, and there was no step on the sagittal view indicating incongruity ( $>$ Fig. 3D).

Magnetic resonance imaging was performed in three dogs (five joints). The sagittal T2-weighted sequence revealed swelling and a hyperintense signal around the oximal aspect of the flexor muscles, being a fluid signal on the fat suppressed STIR sequence ( $>$ Fig. 4 ).

Arthroscopy was performed in all affected joints. At the insertion of the flexor

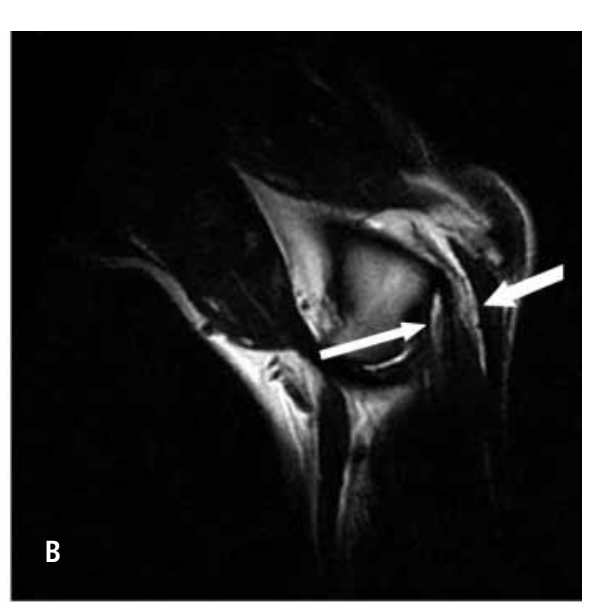

\section{Surgical findings and treatment}

Surgical treatment was performed in three unilateral cases and two dogs had bilateral surgery, with the second surgery being performed one year following the first. Two bilaterally affected dogs had unilateral surgery. One bilateral case was treated with a bilateral injection of corticosteroids $(0.5$ $\mathrm{mg} / \mathrm{kg}$ methylprednisolone), with a period of four years between both joints.

During open surgery, the affected part of the muscle appeared to consist of thickened, hard and white fibrous tissue, located in one muscle or multiple flexor muscles, and which was connected with the surrounding tissue and joint capsule ( $>$ Fig. 6A). After transection, the structure had the aspect of a swollen and partially ruptured, unorganized tendinous tissue (> Fig. 6B).

All dogs returned to complete function following treatment within one to six weeks. According to the owners, the dogs regained full activity and did not need any further medication. Two dogs were not treated on the affected contralateral side and had not shown any clinical problems. On clinical examination the dogs did not show any lameness, the treated joints were 

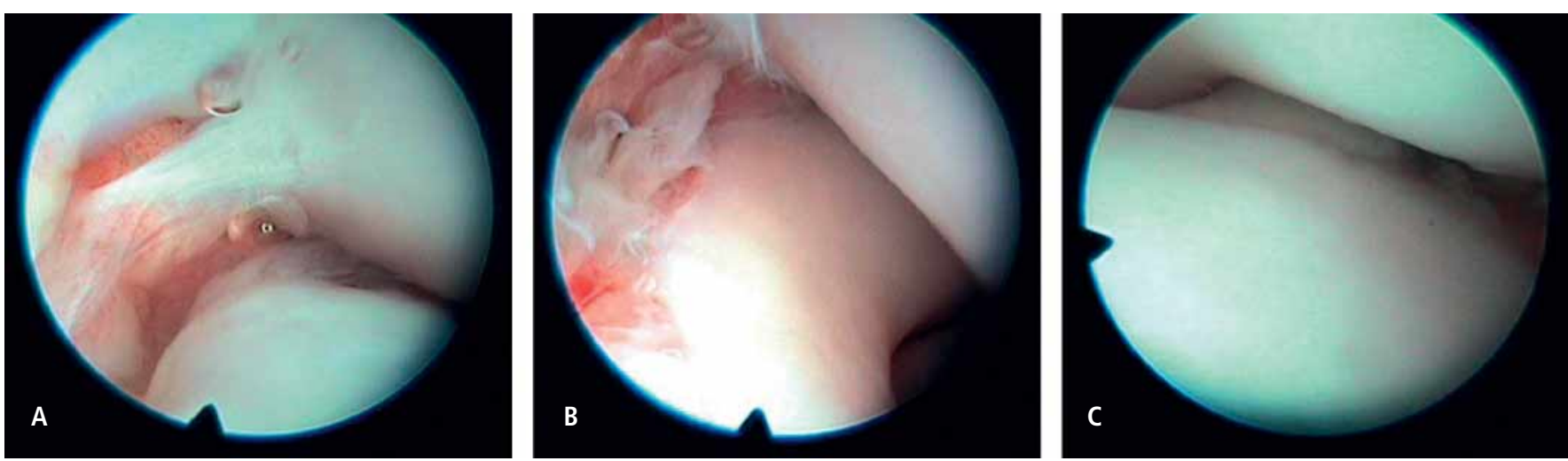

Fig. 5 Arthroscopic views of primary flexor enthesopathy in a 3.75-yearold Great Swiss Mountain dog. A) Segmented and fibrillated aspect of the attachment of the flexor carpi ulnaris muscle, localized synovitis and discrete cartilage erosion. B) Remote view of the attachment of the flexor muscles,

anconeal process and medial aspect of the humeral condyle. C) Intact medial coronoid process; the equal level of the radial head and medial coronoid process suggests congruity of the joint.

not distended and radiography did not show any development of osteoarthritis.

\section{Histopathology}

The excised tissues for the major part consisted of dense collagenous tissue. At the distal end of the samples, there were normal muscle fibres. At the proximal end, synovial villi were present. The collagenous tissue in all cases showed a varying degree of disorganised collagen bundles, presence of young fibroblasts, multiple tortuous small blood vessels and, in two cases, local cartilaginous metaplasia.

\section{Discussion}

This paper draws attention to flexor enthesopathy as a differential diagnosis for elbow lameness with discrete clinical and radiographic changes. Furthermore, flexor enthesopathy is a poorly known elbow disorder and cases with obvious radiographic changes are often considered clinically unimportant. The imaging and arthroscopic findings of eight dogs with primary flexor enthesopathy showing discrete radiographic changes are described. At the initial presentation, the combination of elbow pain and minimal radiographic changes suggested the presumptive diagnosis of a fragmented coronoid process. However, further diagnostic imaging and arthroscopy excluded coronoid problems and instead led to the detection of lesions of the flexor muscles attaching at the medial epicondyle.

In dogs, the 'ununited medial epicondyle' is known as a disorder characterized by the presence of a bony fragment or calcification in the area of the medial epicondyle (6). Since there was no fragment or calcification in the described series, the term 'ununited medial epicondyle' was considered inappropriate. To indicate the disorder, we chose the term 'flexor enthesopathy' adopted from human medicine (19). In human medicine, the 'enthesis', which represents the tendon-to-bone-connection organ, has been receiving great attention lately with enthesitis or enthesopathy being considered as an important cause of locomotion problems. The conditions 'golfers

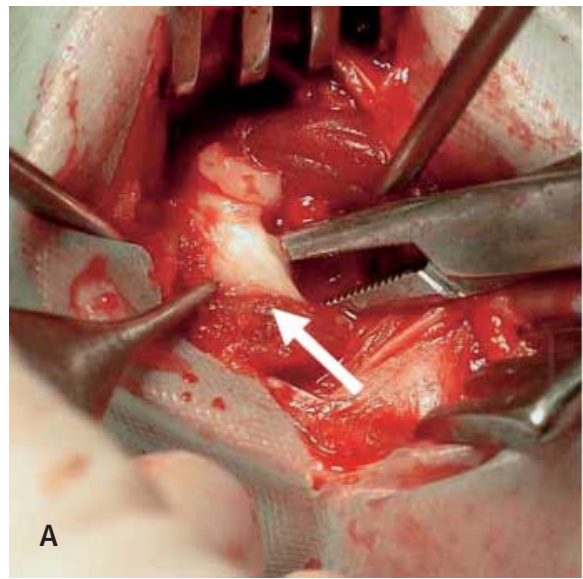

Fig. 6 Aspect of the affected tissue during open surgery. A) White, thickened proximal part of the flexor carpi ulnaris muscle. B) Transection shows the thickened fibrous tissue.

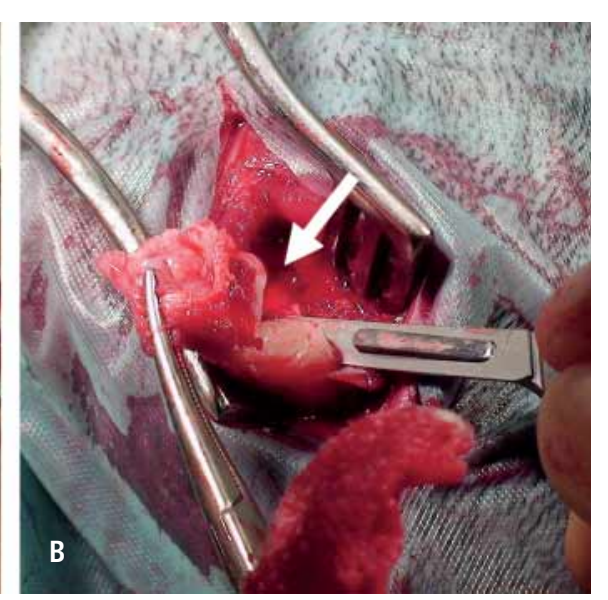

elbow' and 'tennis elbow' are examples of this problem $(18,19)$. In this paper, the term 'primary' is used, because it refers to the absence of underlying pathology in the elbow. This is in contrast with other reports that describe lesions at the medial epicondyle in the presence of fragmented coronoid process and incongruity $(8,12,16,22)$. In those joints, it is unclear whether enthesopathy is the primary cause of the elbow problem or secondary to another elbow disorder. Although several reports have described a radiographically visible fragment or calcification as the cause of lameness, it is often considered as a clinically unimportant finding $(6,22,23)$. Only one report described a 'spur' as the only sign of flexor pathology (17). A spur at the medial epicondyle is often considered as a sign of os-

Vet Comp Orthop Traumatol 3/2012 
teoarthritis and in that case, it is not recognized as a primary problem. However, when a joint is affected by osteoarthritis, osteophytes are spread over the entire joint. In this series, no osteophytes at locations other than the medial epicondyle could be demonstrated except in one case with mild osteoarthritis.

In cases of 'obscure' elbow lameness, it is important to draw attention to primary flexor enthesopathy, in order to consider it in the differential diagnosis when radiographic changes are not obvious. Recent publications attribute obscure elbow lameness to fragmentation of the medial coronoid process, which is often seen as discrete lesions $(2,4,27)$. Recently the term 'medial coronoid disease' was introduced, because in $17.6 \%$ of cases no fragmentation or fissure was found (28). Subtotal coronoidectomy was advised, even in cases where no clear lesion of the medial coronoid process was visible during arthroscopic inspection. Other recently published papers illustrate the difficulty of the diagnosis of medial coronoid disease since many dogs show only minimal clinical and radiographic signs of elbow pathology (2, 4, 29). Since the cases described in this series have a similar profile to dogs affected by fragmented coronoid process or medial coronoid process, a lesion of the flexor tendons should always be considered in the differential diagnosis when 'obscure elbow lameness' is present, in order to make a correct diagnosis and treatment decision $(2,4,28)$.

The age of the dogs in our series varied between two and seven years with a mean of 3.5 years. This is older than the typical age of dogs with medial coronoid disease, which is known as a developmental disorder. However, several recent papers describe the occurrence of medial coronoid disease in older dogs $(2,4,5,28,30)$.

A wide range of imaging techniques were applied in this study. Because radiographic changes were minimal, additional imaging techniques were necessary to demonstrate pathology in the suspected region and to exclude other pathology. Computed tomography enabled the demonstration of bony changes, while arthroscopy confirmed pathology in the area of the medial epicondyle. Both techniques were also helpful in excluding medial coronoid lesions, which was the most probable diagnosis in these cases. Only in a later stage of this series did the authors become more familiar with the disorder and thus decided to apply alternative techniques to enable the visualization of soft tissue structures. This explains why ultrasound and MRI were not used in all cases. High resolution ultrasound enabled excellent visualization of the flexor muscles and their attachment. The bowing, swelling and localized ruptures were easily recognizable. Magnetic resonance imaging also demonstrated swelling and an increased signal that confirmed pathology of the affected muscles. It is possible that not all imaging modalities were necessary to diagnose primary flexor enthesopathy and to distinguish the clinically important lesions from coincidental findings. It was not the object of our study to compare the different imaging modalities for the diagnosis of primary flexor enthesopathy. Moreover the number of patients was too small and this series does not reflect the average presentation. Larger studies including more obvious cases of flexor enthesopathy with visible fragments or calcifications are being performed at our institution in order to define the value of each imaging modality and the clinical significance of each pathological finding.

The diagnosis of primary flexor enthesopathy was thus based on the presence of a variety of specific changes and exclusion of medial coronoid changes by using different imaging modalities. Five contralateral joints were diagnosed as being affected since the same findings were noticed. Although the presence of fluid is the most significant factor to indicate an active process and chronic scar tissue will not necessarily lead to clinical problems, no clear differences could be noticed in this limited series.

Two types of treatment derived from human medicine were performed: intraarticular injection of corticosteroids or surgical removal of the affected tissue (6, 31-33). All dogs became sound after treatment. It was not the intention of this paper to describe and evaluate the most appropriate treatment. Again a larger ongoing study at our hospital should indicate the optimal treatment protocol for flexor enthesopathy.

This paper draws attention to an unrecognized elbow problem, leaving several questions to be answered. It is the author's opinion that primary flexor enthesopathy represents an infrequent yet important option in the differential diagnosis of elbow problems in medium and large breed dogs. Cases of primary enthesopathy may show minimal radiographic changes and hence suggest medial coronoid disease as the cause of lameness. A correct diagnosis of flexor enthesopathy can only be obtained by combining the radiographic findings with other imaging techniques and arthroscopy to confirm suspected lesions of the medial epicondyle and the attaching flexors and to exclude medial coronoid disease.

\section{Conflict of interest \\ None declared.}

\section{References}

1. Kirberger RM, Fourie SL. Elbow dysplasia in the dog: pathophysiology, diagnosis and control. J S Afr Vet Assoc 1998; 69: 43-54.

2. Cook JL, Cook CR. Bilateral shoulder and elbow arthroscopy in dogs with forelimb lameness: diagnostic findings and treatment outcomes. Vet Surg 2009; 38: 224-232.

3. Cook CR, Cook JL. Diagnostic imaging of canine elbow dysplasia: a review. Vet Surg 2009; 38: 144-153.

4. Punke JP, Hulse DA, Kerwin SC, et al. Arthroscopic documentation of elbow cartilage pathology in dogs with clinical lameness without changes on standard radiographic projections. Vet Surg 2009; 38: 209-212.

5. Fitzpatrick N, Smith TJ, Evans RB, et al. Radiographic and arthroscopic findings in the elbow joints of 263 dogs with medial coronoid disease. Vet Surg 2009; 38: 213-223.

6. de Bakker E, Samoy Y, Gielen I, et al. Medial humeral epicondylar lesions in the canine elbow. A review of the literature. Vet Comp Orthop Traumat 2011; 24: 9-17.

7. Ljunggren G, Cawley AJ, Archibald J. The elbow dysplasias in the dog. J Am Vet Med Assoc. 1966; 148: 887-891.

8. Zontine WJ, Weitkamp RA, Lippincott CL. Redefined type of elbow dysplasia involving calcified flexor tendons attached to the medial humeral epicondyle in three dogs. J Am Vet Med Assoc 1989; 194: 1082-1085.

9. Grondalen J, Braut T. Lameness in two young dogs caused by a calcified body in the joint capsule of the elbow. J Small Anim Pract 1976; 17: 681-684.

10. Vaughan LC. Muscle and tendon injuries in dogs. J Small Anim Pract 1979; 20: 711-736.

11. Culvenor JA, Howlett CR. Avulsion of the medial epicondyle of the humerus in the dog. J Smal Anim Pract 1982; 23: 83-89.

12. Walker TM. A redefined type of elbow dysplasia in the dog--2 cases. Can Vet J 1998; 39: 573-575. 
13. Fox SM, Bloomberg MS, Bright RM. Developmental anomalies of the canine elbow. J Am Anim Hosp Assoc 1983; 19: 605-615.

14. Piermattei DL, Flo GL, DeCamp CE. Ununited Medial Epicondyle. In: Brinker, Piermattei and Flo's Handbook of Small Animal Orthopedics and Fracture Repair. 4th ed. Philadelphia: WB Saunders Elsevier; 2006. pg. 352-354.

15. Harasen G. The mysterious dysplastic elbow. Can Vet J 2003; 44: 673-674.

16. Meyer-Lindberg A, Heinen V, Hewicker-Trautwein $\mathrm{M}$, et al. Vorkommen und Behandlung von knochern Metaplasien in den am medialen Epikondylus des Humerus entspringenden Beugesehnen beim Hund [Incidence and treatment of metaplasia in the flexor tendons attached to the medial humeral epicondyle in the dog]. Tierärztl Prax 2004; 32: 276-285.

17. May C, Bennett D. Medial epicondylar spur associated with lameness in dogs. J Small Anim Pract 1988; 29: 797-803

18. Cain EL Jr, Dugas JR, Wolf RS, et al. Elbow injuries in throwing athletes: a current concepts review. Am J Sports Med 2003; 31: 621-635.

19. Benjamin M, Toumi H, Ralphs JR, et al. Where tendons and ligaments meet bone: attachment sites ('entheses') in relation to exercise and/or mechanical load. J Anat 2006; 208: 471-490.
20. Robins G, Innes J. The Elbow. In: Houlton JEF, Cook JL, Innes JF, et al, editors. BSAVA Manual of Canine and Feline Musculoskeletal Disorders. British Small Animal Veterinary Association; 2006. pg. 249-281.

21. Schulz KS, Krotscheck U. In: Textbook of Small Animal Surgery. Philadelphia: Saunders W.B.; 2002. pg. 1927-1952.

22. Paster ER, Biery DN, Lawler DF, et al. Un-united medial epicondyle of the humerus: radiographic prevalence and association with elbow osteoarthritis in a cohort of labrador retrievers. Vet Surg 2009; 38: 169-172.

23. International Elbow Working Group Protocol. Vet Radiol Ultrasound 1995; 36: 172-173.

24. Arkell M, Archer RM, Guitian FJ, et al. Evidence of bias affecting the interpretation of the results of local anaesthetic nerve blocks when assessing lameness in horses. Vet Rec 2006; 159: 346-349.

25. Fuller CJ, Bladon BM, Driver AJ, et al. The intraand inter-assessor reliability of measurement of functional outcome by lameness scoring in horses. Vet J 2006; 171: 281-286.

26. Van Ryssen B, vanBree H, Simoens P. Elbow arthroscopy in clinically normal dogs. Am J Vet Res 1993; 54: 191-198.

27. Smith TJ, Fitzpatrick N, Evans RB, et al. Measurement of ulnar subtrochlear sclerosis using a percentage scale in labrador retrievers with minimal radiographic signs of periarticular osteophytosis. Vet Surg 2009; 38: 199-208.

28. Fitzpatrick N, Smith TJ, Evans RB, et al. Subtotal coronoid ostectomy for treatment of medial coronoid disease in 263 dogs. Vet Surg 2009; 38: 233-245.

29. Fitzpatrick N, Yeadon R. Working algorithm for treatment decision making for developmental disease of the medial compartment of the elbow in dogs. Vet Surg 2009; 38: 285-300.

30. Vermote KA, Bergenhuyzen AL, Gielen I, et al. Elbow lameness in dogs of six years and older: arthroscopic and imaging findings of medial coronoid disease in 51 dogs. Vet Comp Orthop Traumatol 2010; 23: 43-50.

31. Ciccotti MC SMA, Ciccotti MG, Diagnosis and treatment of medial epicondylitis of the elbow. Clin Sports Med. 2004; 23: 693-705.

32. Vangsness CT Jr, Jobe FW. Surgical treatment of medial epicondylitis. Results in 35 elbows. J Bone Joint Surg Br 1991; 73: 409-411.

33. Gabel GT, Morrey BF. Operative treatment of medical epicondylitis. Influence of concomitant ulnar neuropathy at the elbow. J Bone Joint Surg Am 1995; 77: 1065-1069. 